\title{
NR1D1 suppressed the growth of ovarian cancer by abrogating the JAK/STAT3 signaling pathway
}

\author{
Huailin Wang and Yan Fu*
}

\begin{abstract}
Background: Nuclear receptor subfamily 1 group D member 1 (NR1D1), a nuclear receptor associated with a variety of physiological processes, has a low level in ovarian cancer tissues compared with adjacent normal tissues. However, its role in ovarian cancer remains unclear.

Methods: The level of NR1D1 in ovarian cancer cells was determined by quantitative real-time PCR. Its role in ovarian cancer was explored through gain-of-function and lose-of-function. Cell growth was evaluated by CCK8 assay, immunofluorescence and flow cytometry. Western blot was conducted to assess the activation of JAK/STAT3 signaling pathway. A xenograft model of ovarian cancer was established to explore the role of NR1D1 in vivo.

Results: Up-regulation of NR1D1 repressed the ovarian cancer cell proliferation and induced cell cycle arrest and apoptosis, while silencing NR1D1 promoted their proliferation and G1/S transition. In addition, the JAK/STAT3 signaling pathway, an intracellular signal transduction closely associated with cancer progression, was inhibited by NR1D1. Consistently, xenografts with NR1D1 over-expression grew more slowly in vivo than the controls. Furthermore, NR1D1 up-regulated the expression of suppressor of cytokine signaling 3 (SOCS3), an inhibitor of the JAK/STAT3 signaling pathway. Whereas, SOCS3 silencing abolished the function of NR1D1 over-expression on ovarian cancer growth and JAK/STAT3 signaling pathway.

Conclusions: NR1D1 up-regulated the expression of SOCS3, resulting in suppression of the JAK/STAT3 signaling pathway, thus retarding the growth of ovarian cancer cells. This study highlights a profound role of NR1D1 in the treatment of ovarian cancer.
\end{abstract}

Keywords: Ovarian cancer, NR1D1, SOCS3, JAK/STAT3, Proliferation, Apoptosis

\section{Background}

Ovarian cancer is one of the most common gynecologic malignant cancers worldwide. There was an estimate of 22,240 new cases of ovarian cancer and 14,070 deaths in 2018 in the United States [1]. Cytoreductive surgery accompanied with adjuvant chemotherapy is the first-line treatment for ovarian cancer [2]. It leaves an enormous threat to the health care system due to its high

\footnotetext{
* Correspondence: f_y@jlu.edu.cn

Department of Gynecology, the First Hospital of Jilin University, 71 Xinmin Avenue, Changchun 130021, China
}

incidence. Due to the lack of reliable early symptoms, the early diagnosis of ovarian cancer is difficult. Its 5year-survival at distant-stage is nearly $30 \%$ [3]. Thus, it is necessary to have a better understanding of the molecular alterations in ovarian cancer to identify a novel target for early diagnosis and treatment.

Nuclear receptor subfamily 1 group D member 1 (NR1D1), also known as REV-ERB $\alpha$, is a nuclear receptor involved in the regulation of several physiological processes $[4,5]$. NR1D1 is also reported to be involved in cancers. Pharmacological activation of NR1D1 is

(c) The Author(s). 2021 Open Access This article is licensed under a Creative Commons Attribution 4.0 International License, which permits use, sharing, adaptation, distribution and reproduction in any medium or format, as long as you give appropriate credit to the original author(s) and the source, provide a link to the Creative Commons licence, and indicate if changes were made. The images or other third party material in this article are included in the article's Creative Commons licence, unless indicated otherwise in a credit line to the material. If material is not included in the article's Creative Commons licence and your intended use is not permitted by statutory regulation or exceeds the permitted use, you will need to obtain permission directly from the copyright holder. To view a copy of this licence, visit http://creativecommons.org/licenses/by/4.0/ The Creative Commons Public Domain Dedication waiver (http://creativecommons.org/publicdomain/zero/1.0/) applies to the data made available in this article, unless otherwise stated in a credit line to the data. 
lethal to many cancers [6, 7]. It can enhance DNA damage and suppress DNA repair [8, 9]. Additionally, NR1D1 is associated with chemosensitivity of breast cancer [8]. Its high expression has a favorable influence on the survival of patients with breast cancer [10]. According to data from gene expression profiling interactive analysis (GEPIA) website based on the Cancer Genome Atlas (TCGA) data, NR1D1 is lowly expressed in ovarian cancer tissues. However, its role in ovarian cancer remains unclear.

Janus kinase (JAK)/ signal transducer and activator of transcription (STAT) 3 signaling pathway, a famous intracellular signal transduction system, is implicated in various bioprocesses, including proliferation, cycle progress and survival [11]. In response to binding with cytokines or growth factors, these receptors coupled with JAKs undergo a conformational change, leading to the activation of JAKs [12]. Then the JAKs are crossphosphorylated and phosphorylate the cytoplasmic domains of these receptors. Phosphorylated cytoplasmic domains serve as the STAT docking sites, resulting in STAT recruitment and phosphorylation. The phosphorylation of STATs leads to their conformational change and translocation to the nucleus where they perform their function as transcriptional factors regulating the expression of target genes [13]. Abnormal activation of the JAK/STAT3 signaling pathway, which contributes to the pathogenesis and progression of cancers [14-16], was also noted in ovarian cancer $[17,18]$. Suppressing the JAK/STAT3 signal markedly reduces the tumor progression and metastasis of ovarian cancer [19]. Suppressor of cytokine signaling (SOCS) 3, an inhibitor of the JAK/STAT3 signaling pathway, is correlated to the pathogenesis and progression of multiple cancers and regarded as a crucial tumor suppressor [20-22]. SOCS3 obstructs STAT phosphorylation via repressing the activation of JAKs $[23,24]$. Interestingly, the level of SOCS3 was positively correlated to NR1D1 in ovarian cancer according to data from GEPIA. However, whether SOCS3/ JAK/STAT3 is implicated in the role of NR1D1 in ovarian cancer remains not yet clear.

Herein, we explored the function of NR1D1 on the growth of ovarian cancer cells and the activation of SOCS3/JAK/STAT3 signaling pathway. Our results highlight a profound role for NR1D1 in ovarian cancer.

\section{Methods}

\section{Gene expression analysis}

Online website GEPIA (http://gepia.cancer-pku.cn/) was used to analyze the NR1D1 expression in ovarian cancer tissues $(n=426)$ and normal ovarian tissues $(n=88)$ as well as its correlation to SOCS3 based on TCGA data. The Pearson method was employed in the determination of correlation coefficient. Kaplan-Meier plotter databases (https://kmplot.com/) was employed to generate the overall survival curves $(n=1145)$.

\section{Cell culture}

Human ovarian cancer cell lines COC1 (Cat No. CL-0064; Procell, Shanghai, China), A2780 (Cat No. iCell-h004; iCell Bioscience, Shanghai, China) and human normal ovarian epithelial cells (NOEC; Cat No. iCell-h112; iCell Bioscience) were grown in RPMI-1640 (Gibco, Life Technologies, Saint-Aubin, France) with $10 \%$ fetal bovine serum (FBS; Hyclone, Logan, UT, USA). SK-OV-3 cells (Cat No. CL-0215; Procell) were grown in McCoy's 5A (Procell) with 10\% FBS. RPMI-1640 with 20\% FBS was used in the culture of OVCAR-3 cells (Cat No. CL-0178; Procell) and DMEM (Gibco) with 10\% FBS was employed in the culture of $293 \mathrm{~T}$ cells (Cat No. ZQ0033; Zhongqiaoxinzhou Biotechnology, Shanghai, China). All these cells were cultured in a humid atmosphere with $5 \% \mathrm{CO}_{2}$ at $37^{\circ} \mathrm{C}$. All cells were mycoplasma-free and authenticated by short tandem repeat.

\section{Transfection}

Cells seeded in 6 -well plates $\left(4 \times 10^{5}\right.$ cells/well $)$ were transfected with NR1D1 over-expression plasmid (GeneScript, Nanjing, China), NR1D1 shRNAs or SOCS3 siRNAs using Lipofectamine 3000 Reagent (Invitrogen, ThermoFisher, Waltham, Massachusetts, USA) according to the protocol. G418 $(300-400 \mu \mathrm{g} / \mathrm{ml})$ was added into cells for the selection of stably transfected cells. The sequences $\left(5^{\prime}->3^{\prime}\right)$ for NR1D1 shRNAs or SOCS3 siRNAs were as follows:

NR1D1 shRNA-1: GCCCTGAATCCCTCTATAGTTT CAAGAGAACTATAGAGGGATTCAGGGTTTTT; NR1D1 shRNA-2: GGCAACATCACCAAGCTGAATT CAAGAGATTCAGCTTGGTGATGTTGCTTTTT; NR1D1 shRNA-3: GGTCATAACGAGGCCCTAAATT CAAGAGAUTTAGGGCCTCGTTATGACTTTTT; SOCS3-siRNA-1: sense: CCCAGAAGAGCCUA UUACATT; anti-sense: UGUAAUAGGCUCUU CUGGGTT;

SOCS3-siRNA-2: sense: UGGUCACCCACAGC AAGUUTT; anti-sense: AACUUGCUGUGGGUGACC ATT';

SOCS3-siRNA-3: sense: UGGCCACUCUUCAG CAUCUTT; anti-sense: AGAUGCUGAAGAGUGGCC ATT;

Si-NC; sense: UUCUCCGAACGUGUCACGUTT; antisense: ACGUGACACGUUCGGAGAATT.

\section{Quantitative real-time PCR (qRT-PCR)}

A high-purity RNA extraction kit (BioTeke, Beijing, China) was used to extract the total RNA. The first strand of cDNA was synthesized using M-MLV reverse 
transcriptase (TaKaRa Bio, Shiga, Japan). qRT-PCR was performed to determine the mRNA levels of NR1D1 and SOCS3. The primers $\left(5^{\prime}->3^{\prime}\right)$ used were listed below:

NR1D1 forward: CCCTGGGAGTCTACAAGTGG; NR1D1 reverse: GCGATTGATGCGGACGAT; SOCS3 forward: TCGCCACCTACTGAACCCT; SOCS3 reverse: GGTCCAGGAACTCCCGAAT $\beta$-Actin forward: GGCACCCAGCACAATGAA; $\beta$-Actin reverse: TAGAAGCATTTGCGGTGG. $\beta$-Actin served as the internal reference. $2^{-\Delta \Delta C t}$ method was employed to calculate the relative level of target mRNA .

\section{Western blot}

RIPA lysis buffer (with 1\% phenylmethanesulfonyl fluoride) (Beyotime, Shanghai, China) was used to lyse the cells. After determination of protein concentration with a BCA protein concentration determination kit (Beyotime), the protein samples were subjected to sodium dodecyl sulfate-polyacrylamide gel electrophoresis followed by transferation onto polyvinylidene fluoride membranes (ThermoFisher). Following blocking with 5\% bovine serum albumin, the membranes were incubated with antibodies against NR1D1 (1:1000; Abclonal, Wuhan, China), cyclinD (1:1000; ABclonal), cyclinE (1:1000; Proteintech, Wuhan, China), SOCS3 (1:1000; ABclonal), JAK-1 (1:1000; Affinity, Changzhou, China), p-JAK1 (Tyr 1034/Tyr 1035; 1:1000; Affinity), JAK2 (1:500; Affinity), p-JAK2 (Tyr 1007/Tyr 1008, 1:1000; Affinity), STAT3 (1:500; Affinity), p-STAT3 (Tyr 705, 1:500; Affinity), $\beta$-actin (1:2000; Proteintech) at $4{ }^{\circ} \mathrm{C}$ overnight. Thereafter, the membranes were incubated with horseradish peroxidase-labeled secondary antibodies (1:10000; Proteintech) at $37^{\circ} \mathrm{C}$ for $40 \mathrm{~min}$. Blots were visualized with an enhanced chemiluminescence substrate kit (7 Sea biotech, Shanghai, China).

\section{Cell viability assay}

The cell viability was determined by cell counting kit- 8 (CCK-8) (Sigma, St. Louis, MO, USA). Cells seeded in 96-well plates $\left(4 \times 10^{3}\right.$ cells/ well $)$ in quintuplicate were cultured in a cell incubator. At $0 \mathrm{~h}, 6 \mathrm{~h}, 24 \mathrm{~h}, 48 \mathrm{~h}$ and $72 \mathrm{~h}, \mathrm{CCK}-8(10 \mu \mathrm{l})$ was added into cells and incubated for $1 \mathrm{~h}$. A microplate reader (BIOTEK, Winooski, VT, USA) was used to determine the absorbance at $450 \mathrm{~nm}$.

\section{Immunofluorescence}

Paraffin-embedded tumors were cut into $5 \mu \mathrm{m}$-sections. Then the sections were deparaffinated, rehydrated and antigen-retrieved. Cells were seeded onto coverslips. Forty-eight hours later, the cells were fixed in $4 \%$ paraformaldehyde, followed by permeabilizing in $0.1 \%$ TritonX100. Incubation with goat serum (Solarbio, Beijing, China) was performed to block non-specific sites. Then the sections were incubated with antibodies against PCNA (1: 200; Proteintech) or Ki-67(1:100; ABclonal) at $4{ }^{\circ} \mathrm{C}$ overnight, followed by incubating with Cy3-conjudged secondary antibody (1:200; Beyotime). Nucleus was stained with DAPI. Images were captured under a fluorescence microscope (OLYMPUS, Tokyo, Japan).

\section{Flow cytometry}

For cell cycle determination, the cells were fixed in $70 \%$ ice-cold ethanol. After washing in PBS, the cells were stained with propidium iodide and RNaseA in the cell cycle determination kit (Beyotime) at $37^{\circ} \mathrm{C}$ for $30 \mathrm{~min}$. Then the cells were analyzed with a flow cytometry (NovoCyte, ACEA Biosciences, San Diego, CA, USA).

For the determination of cell apoptosis, the cells were stained with a cell apoptosis determination kit (KeyGen, Nanjing, China) at room temperature for $15 \mathrm{~min}$. Then the cells analyzed with a flow cytometry.

\section{Activities of caspase- 3 and caspase-9}

Cells were harvested and lysed. The levels of activated of caspase- 3 and caspase- 9 in cells were determined with a caspase-3 activity determination kit (Beyotime) or caspase- 9 activity determination kit (Solarbio) according to the instructions.

\section{Animal experiment protocols}

$\mathrm{BALB} / \mathrm{c}$ nude mice (4-week-old; Huafukang Bioscience, Beijing, China) were fed in a standard condition (12 hlight/dark cycles, $21-23^{\circ} \mathrm{C}, 45-55 \%$ humidity). $1 \times 10^{6}$ OVCAR-3 cells with stably transfection of NR1D1 overexpression plasmid were subcutaneously injected into the left flank $(n=6)$. Twenty-one days later, the mice were sacrificed and then tumors were harvested for subsequent hematoxylin-eosin (HE) staining, terminal deoxynucleotidyl transferase-mediated dUTP nick end labeling (TUNEL) assay and western blot. All the animal experimental protocols were in accordance with the Guide for Care and Use of Laboratory Animals and approved by the Ethics Committee of the First Hospital of Jilin University (20200698).

\section{HE staining}

Paraffin-embedded tumors were cut into $5 \mu \mathrm{m}$-sections. After deparaffinization and rehydration, the sections were subjected to routine HE staining.

\section{TUNEL assay}

TUNEL assay was performed using an in situ cell death detection kit (Roche, Penzberg, Germany). After deparaffinization and rehydration, the sections were permeabilized in $0.1 \%$ TritonX-100, blocked with $3 \%$ hydrogen peroxide and then stained with TUNEL reaction solution 
in the dark for $1 \mathrm{~h}$. Thereafter, the sections were incubated with Converter-POD solution at $37^{\circ} \mathrm{C}$ for $30 \mathrm{~min}$ and visualized with a DAB substrate kit (Solarbio) and counterstained with hematoxylin. Images were captured under a microscope (OLUMPUS).

\section{Statistical analysis}

Data were presented as mean + standard deviation (SD). Student's t test or one-way analysis of variance followed by Tukey's multiple comparison as the post-hoc were used in data analysis. $P<0.05$ was considered as significant difference.

\section{Results}

\section{NR1D1 inhibited the proliferation of ovarian cancer cells}

To explored the function of NR1D1 in ovarian cancer, we employed GEPIA website to analyze the NR1D1 expression in ovarian cancer tissues $(n=426)$ and normal tissues $(n=88)$. Based on data from TCGA, the GEPIA website showed that NR1D1 expression in ovarian cancer is lower than the normal tissues (Fig. 1A). Additionally, the Kaplan-Meier plotter databases showed that the low NR1D1 expression was associated with poor survival at advanced stages $(n=1145)$ (Fig. 1B). Herein, we investigated the function of NR1D1 in ovarian cancer. First, the NR1D1 level in ovarian cancer cell lines COC1, SKOV3, OVCAR3, A2780 and a normal ovarian epithelial cell NOEC was determined by qRT-PCR and western blot. Ovarian cancer cell lines showed a lower NR1D1 level than NOEC (Fig. 1C-D). Thereafter, a NR1D1 over-expression plasmid was transfected into OVCAR3 cells, which showed the lowest NR1D1 level, to explore the role of NR1D1. In cells transfected with NR1D1 over-expression plasmid, the level of NR1D1 was increased (Fig. 1E). In addition, the proliferation of NR1D1 over-expressed cells was slower than cells transfected with vector (Fig. 1F). The level of PCNA, a biomarker to evaluate the cell proliferation, was also declined in NR1D1 over-expressed cells (Fig. 1G). In addition, shRNAs for NR1D1 were transfected into SK-OV-3 cells, which showed the highest NR1D1 level in ovarian cancer cell lines. NR1D1 shRNAs decreased the level of NR1D1 in ovarian cancer cells (Fig. $1 \mathrm{H}$ ). Meanwhile, the proliferation of NR1D1 silenced cells was accelerated (Fig. 1I), with an increased PCNA level (Fig. 1J). These results suggested that NR1D1 inhibited the proliferation of ovarian cancer cells.

\section{NR1D1 induced cell cycle arrest and apoptosis in ovarian cancer cells}

Cell cycle and apoptosis are important factors impacting the cell growth. The role of NR1D1 in the cell cycle was further explored by flow cytometry. In NR1D1 overexpressed cells, the percentage of cells in G1 phase was increased (Fig. 2A). The levels of cyclinD and cyclinE, which were important factors controlling G1/S transition, were also declined in NR1D1 over-expressed cells (Fig. 2B). In addition, in NR1D1 silenced cells, the percentage of cells in G1 phase was decreased (Fig. 2C), and the levels of cyclinD and cyclinE were increased (Fig. 2D). These results indicated that NR1D1 retarded cell cycle of ovarian cancer cells.

Furthermore, the function of NR1D1 in cell apoptosis was also explored. In NR1D1 over-expressed cells, the percentage of apoptotic cells was increased (Fig. 2E). The activities of caspase- 3 and caspase-9, which were important biomarkers for apoptosis, were also increased in NR1D1 over-expressed cells (Fig. 2F). These results suggested that NR1D1 induced apoptosis of ovarian cancer cells.

\section{NR1D1 inhibited the activation of JAK/STAT3 signaling pathway}

The JAK/STAT3 signaling pathway plays a critical role in cancers. We found that in NR1D1 over-expressed cells, the levels of phosphorylated JAK1, JAK2 and STAT3 were decreased, while there were no significant changes in the levels of total JAK1, JAK2 and STAT3 (Fig. 3A). Consistently, in NR1D1 silenced cells, the levels of phosphorylated JAK1, JAK2 and STAT3 were increased, with no significant changes in the levels of total JAK1, JAK2 and STAT3 (Fig. 3B). These results revealed that NR1D1 inhibited the activation of JAK/ STAT3 signaling pathway.

\section{NR1D1 suppressed the growth of ovarian cancer cells} in vivo

The role of NR1D1 in the growth of ovarian cancer cells was also explored in vivo. Tumors with NR1D1 over-expression were smaller than the controls (vector), with lighter tumor weights (Fig. 4A). Tumors with NR1D1 over-expression showed increased TUNEL staining with distinct regions of apoptosis/necrosis unlike tumors in the control group (Fig. $4 \mathrm{~B})$, which was consistent with the decreased tumor volumes. Besides, as determined by immunofluorescence, there was a decreased Ki-67 staining in NR1D1 over-expressed tumors compared with the controls (Fig. 4C). Furthermore, in tumors with NR1D1 over-expression, the levels of phosphorylated JAK1, JAK2 and STAT3 were decreased, whereas, the level of total JAK1, JAK2 and STAT3 showed no significant difference (Fig. 4D). These results suggested that NR1D1 suppressed the in vivo growth of ovarian cancer cells and activation of the JAK/STAT3 signaling pathway.

\section{NR1D1 inhibited the proliferation and induces apoptosis through up-regulating SOCS3}

The level of SOCS3 in ovarian cancer cell lines was lower than that in NOEC, at both mRNA level and protein level (Fig. 5A-B). According to data from TCGA, 


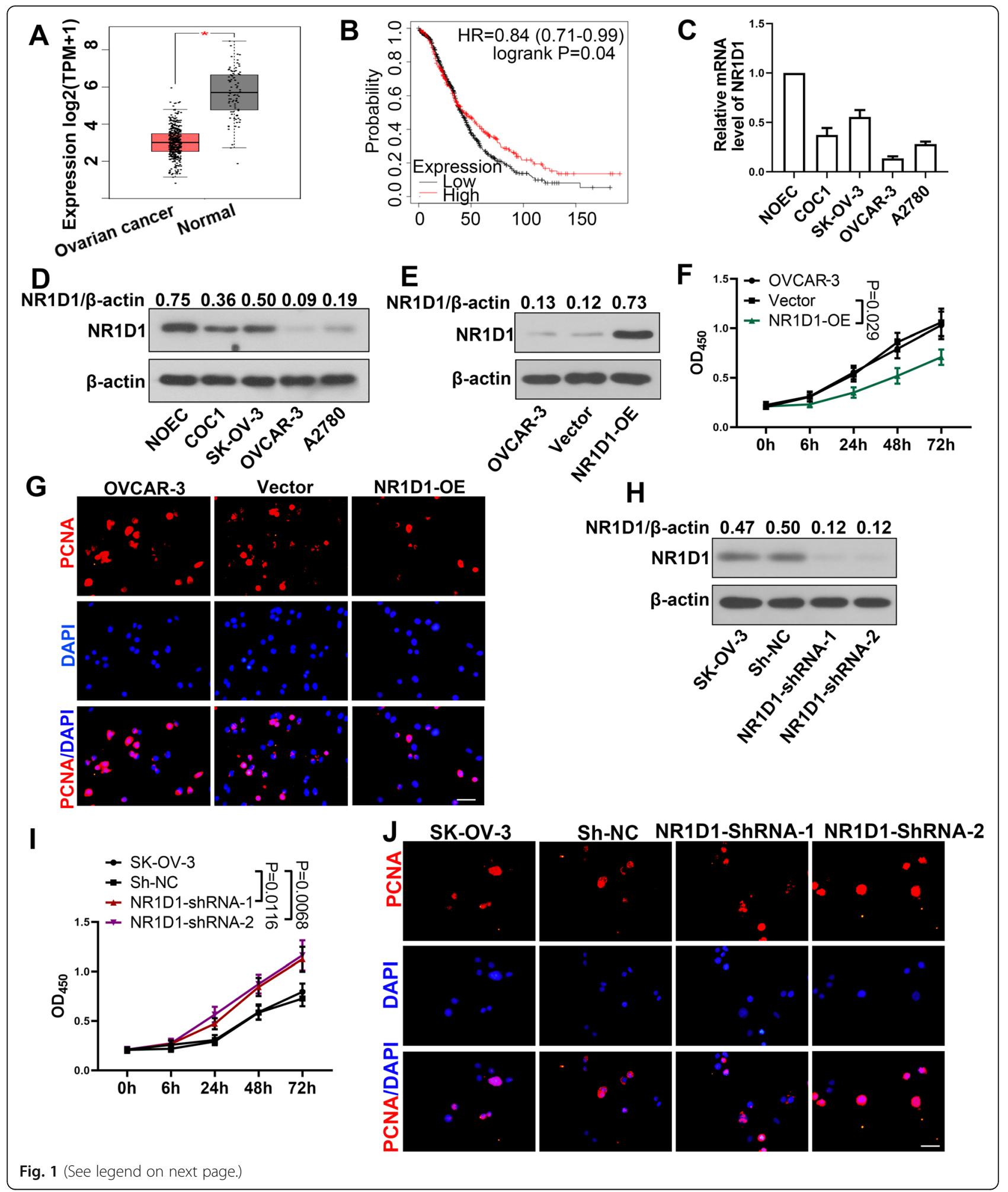


(See figure on previous page.)

Fig. 1 NR1D1 inhibited the proliferation of ovarian cancer cells. A Data from TCGA showed that NR1D1 has a low expression level in ovarian cancer tissues (ovarian cancer $n=426$ and normal ovarian tissues $n=88$ ). B Low NR1D1 level was correlated to the poor survival of ovarian cancer patients at advanced stage $(n=1145)$. C-D The level of NR1D1 in ovarian cancer cell lines COC1, SK-OV-3, OVCAR-3, A2780 and normal ovarian epithelial cell line (NOEC) was determined by quantitative real-time PCR and western blot. E Transfection efficiency of NR1D1 over-expression plasmid was determined by western blot. F After transfection with NR1D1 over-expression plasmid, the proliferation of ovarian cancer cells was determined with CCK-8. G The level of PCNA in ovarian cancer cells was determined by immunofluorescence after transfection with NR1D1 over-expression plasmid. Scale bar = $50 \mu \mathrm{m}$. H The transfection efficiencies of NR1D1 shRNAs in ovarian cancer cells were determined by western blot. I The proliferation of NR1D1 silenced cells was determined with CCK-8. (J) Immunofluorescence was performed to determine the level of PCNA in NR1D1 silenced cells. Scale bar $=50 \mu \mathrm{m}$. The results are presented as mean \pm SD

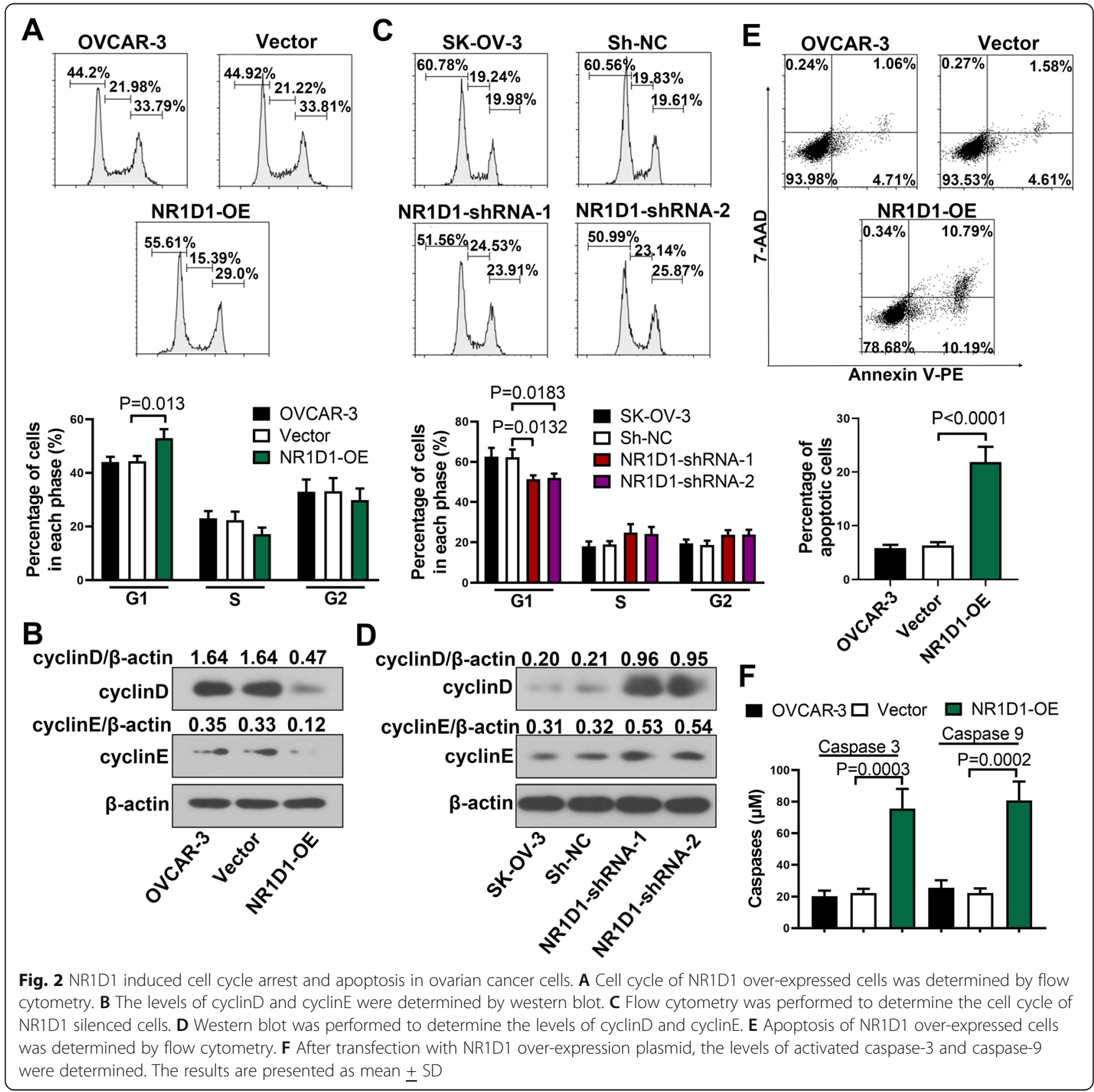




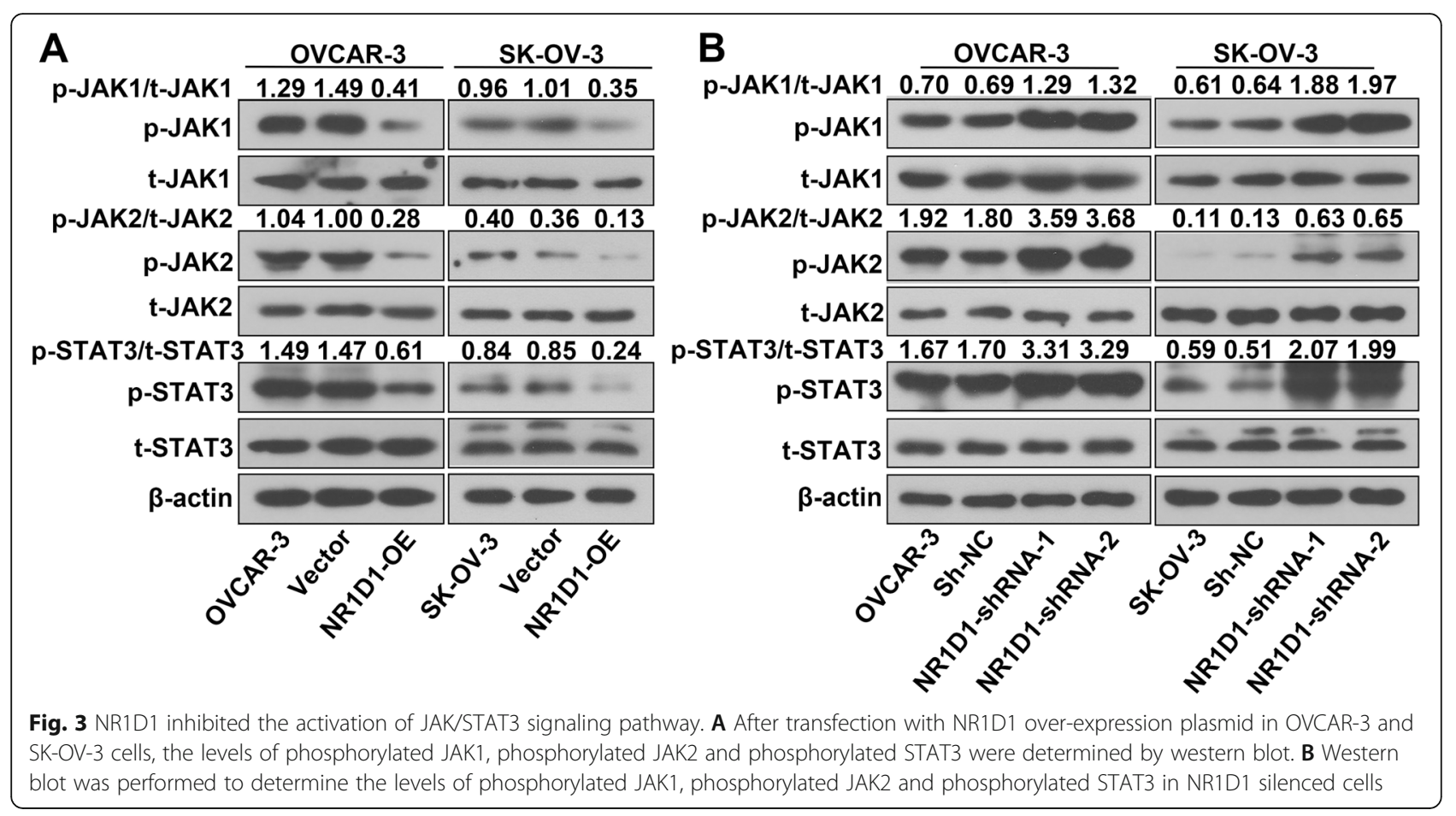

NR1D1 was positively correlated with SOCS3, an inhibitor of the JAK/STAT3 signaling pathway (Fig. 5C). In NR1D1 over-expressed cells, the level of SOCS3 was increased, while in NR1D1 silenced cells, the SOCS3 level was decreased (Fig. 5D-F). On the other hand, in tumors with NR1D1 over-expressed, the level of SOCS3 was also increased (Fig. 5G). These results demonstrated that NR1D1 positively regulated the expression of SOCS3.

Thereafter, SOCS3 siRNAs were introduced to explore whether NR1D1 performed its function through regulating SOCS3. First, the efficiencies of SOCS3 siRNAs were confirmed by western blot (Fig. $5 \mathrm{H}$ ). Thereafter, SOCS3 siRNA was co-transfected into cells with NR1D1 overexpression, and then the cell viability, apoptosis and phosphorylation of STAT3 were detected. In cells cotransfected with NR1D1 over-expression plasmid and SOCS3 siRNA, the cell viability was higher (Fig. 5I) and the percentage of apoptotic cells was lower (Fig. 5J) than cells co-transfected with NR1D1 over-expression plasmid and si-NC. In addition, the level of phosphorylated STAT3 was increased in cells co-transfected with NR1D1 over-expression plasmid and SOCS3 siRNA (Fig. $5 \mathrm{~K})$. It was indicated that silencing SOCS3 abolished the function of NR1D1 over-expression on the proliferation and apoptosis of ovarian cancer cells as well as the activation of STAT3 signal (Fig. 6).

\section{Discussion}

Data from TCGA showed that NR1D1 has a low expression level in ovarian cancer tissues, whereas, its role in ovarian cancer is not yet clear. In the present study, we explored the function of NR1D1 in ovarian cancer. Our study showed that NR1D1 suppressed the proliferation and induced apoptosis of ovarian cancer cells. Further study showed that NR1D1 inhibited the activation of JAK/ STAT3 signaling pathway through positively regulating SOCS3. These data highlight a profound role of NR1D1 in the treatment of ovarian cancer. NR1D1 is also implicated in the regulation of circadian rhythm. Circadian rhythm disruption is reported to increase the cancer risk [25, 26]. Accordingly, the World Health Organization classified the shift-work, which was associated with disrupted circadian rhythm, as a possible carcinogen [27]. Moreover, circadian rhythm gene Bmal1, which was down-regulated in tongue squamous cell carcinoma (TSCC), suppressed the growth and metastasis of TSCC [28]. Circadian rhythm gene PER2, which had a low expression in ovarian cancer cells, repressed the growth of ovarian cancer cell SKOV-3 and enhanced their sensitivity to cisplatin [29]. Hence, abnormal expression of circadian rhythm genes has a close relationship with cancer risk and chemotherapy sensitivity [29-33].

Dysregulation of gene expression in cancers may contribute to the tumourgenesis. NR1D1 has a low level in ovarian cancer cell lines. Also, according to data from TCGA, NR1D1 has a low level in ovarian cancer tissues. Its low level is also associated with the poor survival of ovarian cancer patients at advanced stage. Hence, we wonder what the function of NR1D1 is in ovarian cancer cells. Over-expression of NR1D1 retarded the 


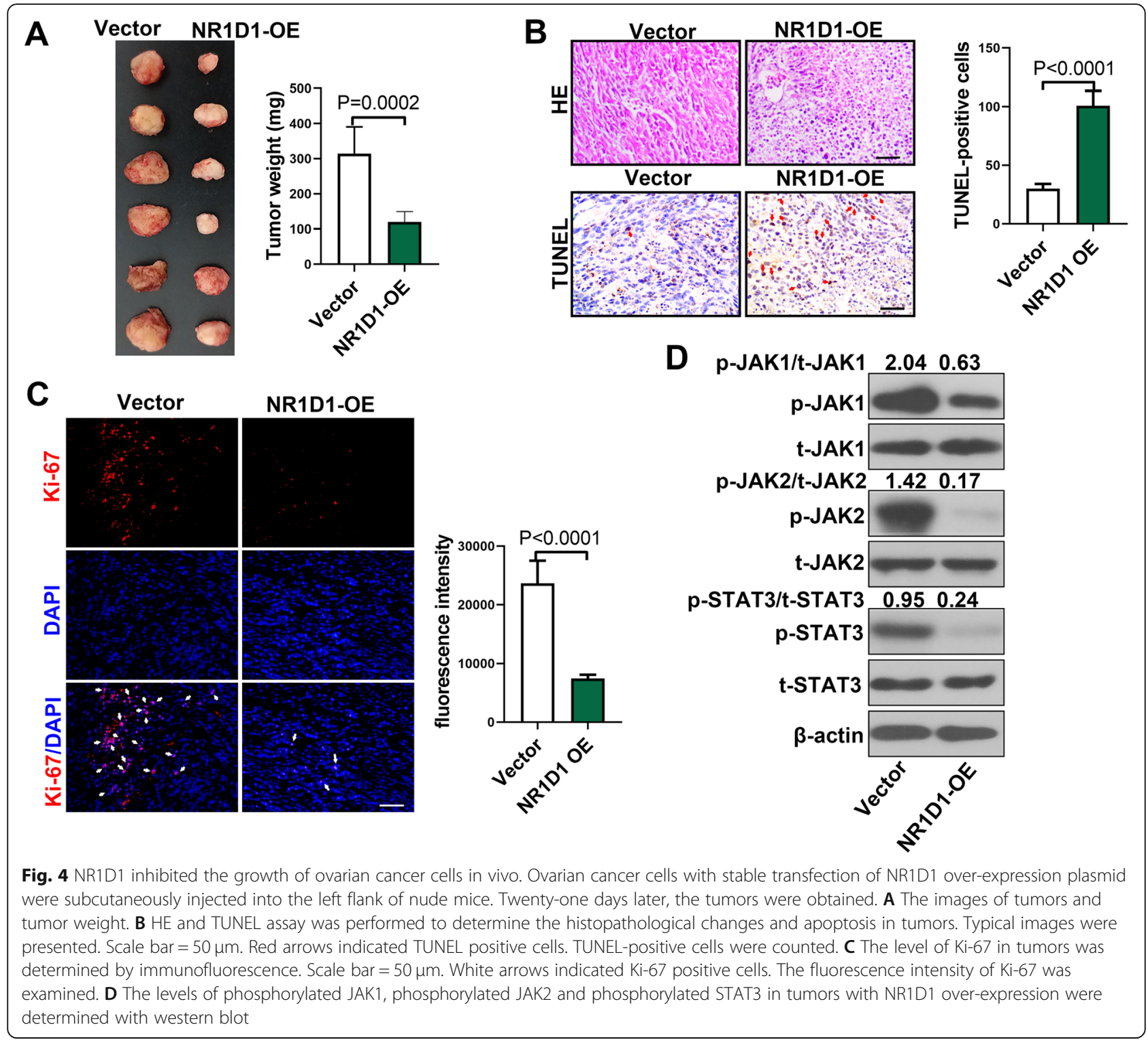

proliferation of ovarian cancer cells, while NR1D1 silencing accelerated their growth, suggesting that NR1D1 suppresses the growth of ovarian cancer cells. In addition, PCNA, which is closely related to cell proliferation and served as a biomarker of cell growth, was also reduced by NR1D1. These results provided additional evidence for our hypothesis that NR1D1 suppresses the growth of ovarian cancer. Besides, its in vivo growth was also repressed by over-expression of NR1D1, indicating that NR1D1 may act as a tumor suppressor. Consistently, down-regulation of NR1D1 promotes the proliferation of colon cancer cells [34], while activation of NR1D1 is lethal to cancer cells [6]. NR1D1 is also associated chemosensitivity [8]. Hence, we hypothesize that activation of NR1D1 may be beneficial to the treatment of ovarian cancer.
Cell cycle is a key factor that orchestrates the cell growth. Our study showed that NR1D1 arrested cell cycle at G1 phase and decreased the levels cell cycleassociated proteins, such as cyclins, indicating that cell cycle arrest induced by NR1D1 may contribute to its role in ovarian cancer growth. Consistently, activation of NR1D1 was also reported to reduce the level of cyclinA in breast cancer cells [7]. On the other hand, apoptosis was induced by NR1D1 over-expression, which may contribute to the tumor-suppressor role of NR1D1 in ovarian cancer. Apoptosis of cancer cells contributes to the therapeutic effects of anti-tumor drugs. NR1D1 is reported to be recruited to the damaged DNAs and inhibits their repair [8]. It also enhances the accumulation of ROS-induced DNA damage in breast cancer cells through the interaction with PARP1, thus increasing 


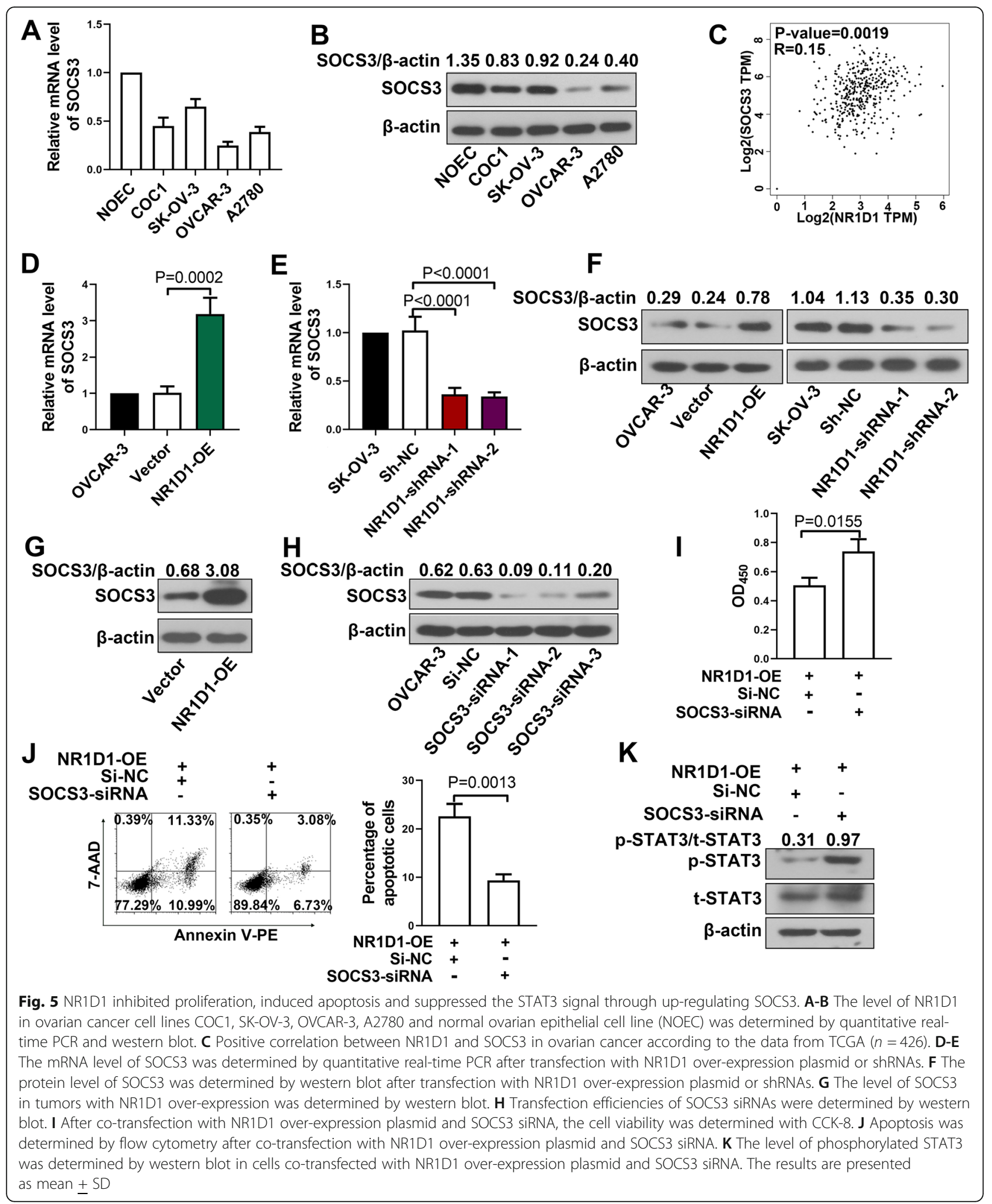

their sensitivity to oxidative stress [9]. As chemotherapeutic drugs for ovarian cancer treatment also promote the generation of ROS [35], we speculate that NR1D1 may also influence the chemoresistance of ovarian cancer cells. This speculation may be further confirmed in our future study. 


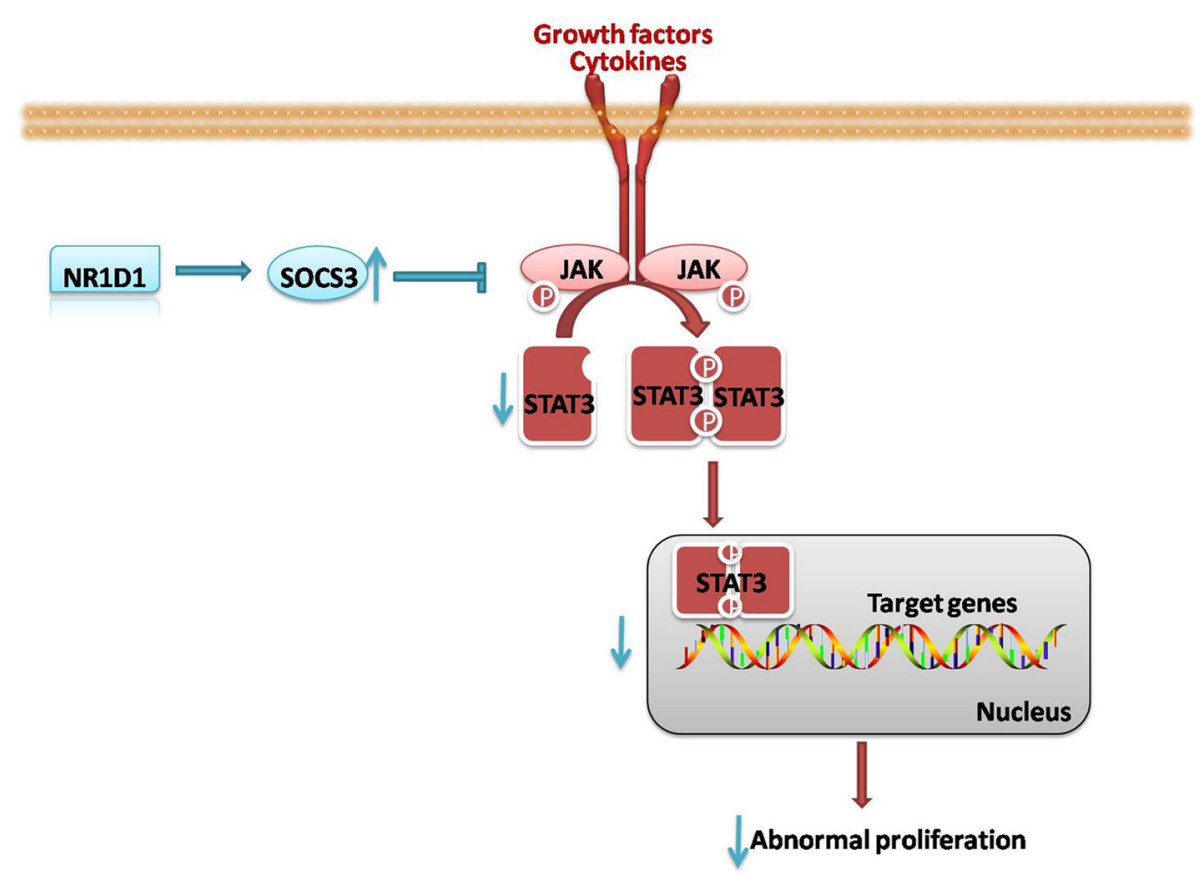

Fig. 6 Schematic diagram: NR1D1 suppressed the growth of ovarian cancer through the JAKJSTAT3 signaling pathway

Activation of the JAK/STAT3 signaling pathway contributes to the uncontrolled proliferation of cancer cells, including ovarian cancer [36]. Inhibition of the JAK/ STAT3 signal suppresses the growth of ovarian cancer cells [37] and reduces their dissemination to the peritoneal cavity [19]. In our study, the JAK/STAT3 signaling pathway was inhibited by NR1D1 over-expression and enhanced by NR1D1 silencing, indicating that the JAK/ STAT3 signal may be implicated in the role of NR1D1 in ovarian cancer cells. We wonder how NR1D1 influences the JAK/STAT3 signal in ovarian cancer cells. SOCS3 is an inhibitor of the JAK/STAT3 signaling pathway. The expression of SOCS3 is induced by excessive STATs activation, which in turn suppresses the bond of JAKs to receptors, thus suppressing their kinase activities as well as the phosphorylation of STAT3 [38]. On the other hand, SOCS3 promotes the ubiquitination and degradation of JAKs, thus reducing their stability [39]. As SOCS3 has a low expression level in ovarian cancer tissues [40] and positively correlated with the level of NR1D1, we speculate that NR1D1 may influence the JAK/STAT3 signaling pathway as well as ovarian cancer cell growth through modulating SOCS3. We found that NR1D1 positively regulated the expression of SOCS3. Additionally, silencing SOCS3 abolished the effect of NR1D1 over-expression on the proliferation and apoptosis of ovarian cancer cells, confirming that NR1D1 performs its role in ovarian cancer cells through modulating the expression of SOCS3. However, it is unclear how NR1D1 regulates the level of SOCS3. According to literature review, NR1D1 positively regulated the expression of transcription factor EB (TFEB) [41], while TFEB up-regulated the expression of SOCS3 [42]. Thus we speculated that NR1D1 may up-regulate the level of SOCS3 via TFEB. Other cytokines may also participate in the regulation of SOCS3 by NR1D1, and more researches are needed.

\section{Conclusions}

Our study revealed that NR1D1 inhibited the activation of JAK/STAT3 signaling pathway through up-regulating SOCS3, thus suppressing proliferation and inducing apoptosis of ovarian cancer cells. These results indicate that NR1D1 may act as a tumor-suppressor in ovarian cancer cells, and provide basis for novel strategy of ovarian cancer treatment.

\section{Abbreviations}

NR1D1: Nuclear receptor subfamily 1 group D member 1; JAK: Janus kinase: STAT: Signal transducer and activator of transcription; SOCS3: Suppressor of cytokine signaling 3; TCGA: The Cancer Genome Atlas; FBS: Fetal bovine serum; PCNA: Proliferating cell nuclear antigen; HE: Hematoxylin-eosin; TFEB: Transcription factor EB; TUNEL: Terminal deoxynucleotidyl transferasemediated dUTP nick end labeling; SD: Standard deviation

\section{Supplementary Information}

The online version contains supplementary material available at https://doi. org/10.1186/s12885-021-08597-8.

Additional file 1. Original blot images. 


\section{Acknowledgements}

Not applicable.

\section{Authors' contributions}

HW and YF designed this study, analyzed the data and wrote the manuscript; HW performed the experiments. All authors have read and approved the final manuscript.

\section{Funding}

This research was funded by the National Natural Science Foundation of China (No. 81671574 and No. 81470975). The funding bodies played no role in the design of the study and collection, analysis, and interpretation of data and in writing the manuscript.

\section{Availability of data and materials}

All data generated or analyzed during this study are included in this article.

\section{Declarations}

\section{Ethics approval and consent to participate}

All animal experiments involved in this study were approved by the Ethics Committee of the First Hospital of Jilin University (20200698).

\section{Consent for publication}

Not applicable.

\section{Competing interests}

The authors declare that they have no competing interests.

Received: 17 January 2021 Accepted: 6 July 2021

Published online: 30 July 2021

\section{References}

1. Torre LA, Trabert B, DeSantis CE, Miller KD, Samimi G, Runowicz CD, et al. Ovarian cancer statistics, 2018. CA Cancer J Clin. 2018;68(4):284-96. https:// doi.org/10.3322/caac.21456.

2. Kuroki L, Guntupalli SR. Treatment of epithelial ovarian cancer. BMJ. 2020; 371:m3773.

3. Siegel RL, Miller KD, Jemal A. Cancer statistics, 2020. CA Cancer J Clin. 2020; 70(1):7-30.

4. Duez H, Staels B. Rev-erb-alpha: an integrator of circadian rhythms and metabolism. J Appl Physiol (1985). 2009;107(6):1972-80.

5. Everett LJ, Lazar MA. Nuclear receptor rev-erbalpha: up, down, and all around. Trends Endocrinol Metab. 2014;25(11):586-92. https://doi.org/10.101 6/j.tem.2014.06.011.

6. Sulli G, Rommel A, Wang X, Kolar MJ, Puca F, Saghatelian A, et al. Pharmacological activation of REV-ERBs is lethal in cancer and oncogeneinduced senescence. Nature. 2018;553(7688):351-5. https://doi.org/10.1038/ nature25170.

7. Wang Y, Kojetin D, Burris TP. Anti-proliferative actions of a synthetic REVERBalpha/beta agonist in breast cancer cells. Biochem Pharmacol. 2015. 96(4):315-22. https://doi.org/10.1016/j.bcp.2015.06.010.

8. Ka NL, Na TY, Na H, Lee MH, Park HS, Hwang S, et al. NR1D1 recruitment to sites of DNA damage inhibits repair and is associated with Chemosensitivity of breast Cancer. Cancer Res. 2017;77(9):2453-63. https://doi.org/10.1158/ 0008-5472.CAN-16-2099.

9. Ka NL, Na TY, Lee MO. NR1D1 enhances oxidative DNA damage by inhibiting PARP1 activity. Mol Cell Endocrinol. 2017;454:87-92. https://doi. org/10.1016/j.mce.2017.06.004.

10. $\mathrm{Na} \mathrm{H}$, Han J, Ka NL, Lee MH, Choi YL, Shin YK, et al. High expression of NR1D1 is associated with good prognosis in triple-negative breast cancer patients treated with chemotherapy. Breast Cancer Res. 2019;21(1):127. https://doi.org/10.1186/s13058-019-1197-x

11. Groner B, von Manstein V. Jak Stat signaling and cancer: opportunities, benefits and side effects of targeted inhibition. Mol Cell Endocrinol. 2017; 451:1-14. https://doi.org/10.1016/j.mce.2017.05.033

12. Brooks AJ, Dai W, O'Mara ML, Abankwa D, Chhabra Y, Pelekanos RA, et al. Mechanism of activation of protein kinase JAK2 by the growth hormone receptor. Science. 2014;344(6185):1249783. https://doi.org/10.1126/ science.1249783.
13. Leonard WJ, O'Shea JJ. Jaks and STATs: biological implications. Annu Rev Immunol. 1998;16(1):293-322. https://doi.org/10.1146/annurev.immunol.1 6.1.293.

14. Li HX, Zhao W, Shi Y, Li YN, Zhang LS, Zhang HQ, et al. Retinoic acid amide inhibits JAKVSTAT pathway in lung cancer which leads to apoptosis. Tumour Biol. 2015;36(11):8671-8. https://doi.org/10.1007/s13277-015-3534-8.

15. Johnson DE, O'Keefe RA, Grandis JR. Targeting the IL-6/JAK/STAT3 signalling axis in cancer. Nat Rev Clin Oncol. 2018;15(4):234-48. https://doi.org/10.103 8/nrclinonc.2018.8.

16. Banerjee $\mathrm{K}$, Resat $\mathrm{H}$. Constitutive activation of STAT3 in breast cancer cells: a review. Int J Cancer. 2016;138(11):2570-8. https://doi.org/10.1002/ijc.29923.

17. Wen W, Han ES, Dellinger TH, Wu J, Guo Y, Buettner R, et al. Increasing antitumor activity of JAK inhibitor by simultaneous blocking multiple survival signaling pathways in human ovarian Cancer. Transl Oncol. 2019; 12(8):1015-25. https://doi.org/10.1016/j.tranon.2019.05.003.

18. Chen M, Zeng J, Chen S, Li J, Wu H, Dong X, et al. SPTBN1 suppresses the progression of epithelial ovarian cancer via SOCS3-mediated blockade of the JAK/STAT3 signaling pathway. Aging (Albany NY). 2020;12(11):10896911. https://doi.org/10.18632/aging.103303.

19. Wen W, Liang W, Wu J, Kowolik CM, Buettner R, Scuto A, et al. Targeting JAK1/STAT3 signaling suppresses tumor progression and metastasis in a peritoneal model of human ovarian cancer. Mol Cancer Ther. 2014;13(12): 3037-48. https://doi.org/10.1158/1535-7163.MCT-14-0077.

20. Huang L, Hu B, Ni J, Wu J, Jiang W, Chen C, et al. Transcriptional repression of SOCS3 mediated by IL-6/STAT3 signaling via DNMT1 promotes pancreatic cancer growth and metastasis. J Exp Clin Cancer Res. 2016;35(1): 27. https://doi.org/10.1186/s13046-016-0301-7.

21. Wu WY, Kim H, Zhang CL, Meng XL, Wu ZS. Loss of suppressors of cytokine signaling 3 promotes aggressiveness in hepatocellular carcinoma. J Investig Surg. 2014;27(4):197-204. https://doi.org/10.3109/08941939.2013.873098.

22. Wan J, Che Y, Kang N, Wu W. SOCS3 blocks HIF-1alpha expression to inhibit proliferation and angiogenesis of human small cell lung cancer by downregulating activation of Akt, but not STAT3. Mol Med Rep. 2015;12(1): 83-92. https://doi.org/10.3892/mmr.2015.3368.

23. Tamiya T, Kashiwagi I, Takahashi R, Yasukawa H, Yoshimura A. Suppressors of cytokine signaling (SOCS) proteins and JAK/STAT pathways: regulation of T-cell inflammation by SOCS1 and SOCS3. Arterioscler Thromb Vasc Biol. 2011;31(5):980-5. https://doi.org/10.1161/ATVBAHA.110.207464.

24. Durham GA, Williams JJL, Nasim MT, Palmer TM. Targeting SOCS proteins to control JAK-STAT Signalling in disease. Trends Pharmacol Sci. 2019;40(5): 298-308. https://doi.org/10.1016/j.tips.2019.03.001.

25. Filipski E, King VM, Li X, Granda TG, Mormont MC, Liu X, et al. Host circadian clock as a control point in tumor progression. J Natl Cancer Inst. 2002;94(9): 690-7. https://doi.org/10.1093/jnci/94.9.690.

26. Aiello I, Fedele MLM, Roman F, Marpegan L, Caldart C, Chiesa JJ, et al. Circadian disruption promotes tumor-immune microenvironment remodeling favoring tumor cell proliferation. Sci Adv. 2020;6(42):eaaz4530.

27. Straif K, Baan R, Grosse Y, Secretan B, El Ghissassi F, Bouvard V, et al. Carcinogenicity of shift-work, painting, and fire-fighting. Lancet Oncol. 2007; 8(12):1065-6. https://doi.org/10.1016/S1470-2045(07)70373-X.

28. Tang Q, Cheng B, Xie M, Chen Y, Zhao J, Zhou X, et al. Circadian clock gene Bmal1 inhibits tumorigenesis and increases paclitaxel sensitivity in tongue squamous cell carcinoma. Cancer Res. 2017;77(2):532-44. https://doi.org/1 0.1158/0008-5472.CAN-16-1322.

29. Wang Z, Li F, Wei M, Zhang S, Wang T. Circadian clock protein PERIOD2 suppresses the PI3K/Akt pathway and promotes cisplatin sensitivity in ovarian Cancer. Cancer Manag Res. 2020;12:11897-908. https://doi.org/1 0.2147/CMAR.S278903.

30. Shostak A. Circadian clock, cell division, and Cancer: from molecules to organism. Int J Mol Sci. 2017;18(4):873. https://doi.org/10.3390/ijms1804 0873.

31. Wang Z, Wang H, Guo H, Li F, Wu W, Zhang S, et al. The circadian rhythm and core gene Period 2 regulate the chemotherapy effect and multidrug resistance of ovarian cancer through the PI3K signaling pathway. Biosci Rep. 2020;40(11):BSR20202683. https://doi.org/10.1042/BSR20202683.

32. Jim HS, Lin HY, Tyrer JP, Lawrenson K, Dennis J, Chornokur G, et al. Common genetic variation in circadian rhythm genes and risk of epithelial ovarian Cancer (EOC). J Genet Genome Res. 2015;2(2):017.

33. Carter BD, Diver WR, Hildebrand JS, Patel AV, Gapstur SM. Circadian disruption and fatal ovarian cancer. Am J Prev Med. 2014:46(3 Suppl 1):S3441. https://doi.org/10.1016/j.amepre.2013.10.032. 
34. Basti A, Fior R, Yalin M, Povoa V, Astaburuaga R, Li Y, et al. The Core-clock gene NR1D1 impacts cell motility in vitro and invasiveness in a zebrafish xenograft Colon Cancer model. Cancers (Basel). 2020;12(4):853. https://doi. org/10.3390/cancers 12040853.

35. Santiago-O'Farrill JM, Weroha SJ, Hou X, Oberg AL, Heinzen EP, Maurer MJ, et al. Poly (adenosine diphosphate ribose) polymerase inhibitors induce autophagy-mediated drug resistance in ovarian cancer cells, xenografts, and patient-derived xenograft models. Cancer. 2020;126(4):894-907. https://doi. org/10.1002/cncr.32600.

36. Rosen DG, Mercado-Uribe I, Yang G, Bast RC Jr, Amin HM, Lai R, et al. The role of constitutively active signal transducer and activator of transcription 3 in ovarian tumorigenesis and prognosis. Cancer. 2006;107(11):2730-40. https://doi.org/10.1002/cncr.22293.

37. Gritsina G, Xiao F, O'Brien SW, Gabbasov R, Maglaty MA, Xu RH, et al. Targeted blockade of JAK/STAT3 signaling inhibits ovarian carcinoma growth. Mol Cancer Ther. 2015;14(4):1035-47. https://doi.org/10.1158/15357163.MCT-14-0800.

38. Babon JJ, Nicola NA. The biology and mechanism of action of suppressor of cytokine signaling 3. Growth Factors. 2012;30(4):207-19. https://doi.org/1 0.3109/08977194.2012.687375.

39. Linossi EM, Calleja DJ, Nicholson SE. Understanding SOCS protein specificity. Growth Factors. 2018;36(3-4):104-17. https://doi.org/10.1080/08977194.201 8.1518324

40. Shang $A Q$, Wu J, Bi F, Zhang YJ, Xu LR, Li LL, et al. Relationship between HER2 and JAK/STAT-SOCS3 signaling pathway and clinicopathological features and prognosis of ovarian cancer. Cancer Biol Ther. 2017;18(5):31422. https://doi.org/10.1080/15384047.2017.1310343.

41. Chandra V, Bhagyaraj E, Nanduri R, Ahuja N, Gupta P. NR1D1 ameliorates mycobacterium tuberculosis clearance through regulation of autophagy. Autophagy. 2015;11(11):1987-97. https://doi.org/10.1080/15548627.2015.1 091140.

42. Li Y, Hodge J, Liu Q, Wang J, Wang Y, Evans TD, et al. TFEB is a master regulator of tumor-associated macrophages in breast cancer. J Immunother Cancer. 2020;8(1):e000543. https://doi.org/10.1136/jitc-2020-000543.

\section{Publisher's Note}

Springer Nature remains neutral with regard to jurisdictional claims in published maps and institutional affiliations.

Ready to submit your research? Choose BMC and benefit from:

- fast, convenient online submission

- thorough peer review by experienced researchers in your field

- rapid publication on acceptance

- support for research data, including large and complex data types

- gold Open Access which fosters wider collaboration and increased citations

- maximum visibility for your research: over $100 \mathrm{M}$ website views per year

At $\mathrm{BMC}$, research is always in progress.

Learn more biomedcentral.com/submissions 